\title{
Primary Care Providers' Perceptions About Participating in Low-Risk Prostate Cancer Treatment Decisions
}

\author{
Archana Radhakrishnan, $M D, M H S^{7}$ id, Lauren P. Wallner, $P h D, M P H^{1,2}$, \\ Ted A. Skolarus, MD, MPH, FACS ${ }^{3,4}$, Paul H. Abrahamse, MA ${ }^{7}$, Adam S. Kollipara' \\ Steven J. Katz, MD, MPH ${ }^{1,5}$, and Sarah T. Hawley, PhD, MPH $H^{1,4,5}$
}

'Department of Internal Medicine, University of Michigan, Ann Arbor, MI, USA; ${ }^{2}$ Department of Epidemiology, University of Michigan, Ann Arbor, MI, USA; ${ }^{3}$ Department of Urology, University of Michigan, Ann Arbor, MI, USA; ${ }^{4}$ Ann Arbor VA Center for Clinical Management Research, Ann Arbor, MI, USA; ${ }^{5}$ Department of Health Management and Policy, University of Michigan, Ann Arbor, MI, USA.

BACKGROUND: Primary care provider's (PCP) role in cancer care is expanding and may include supporting patients in their treatment decisions. However, the degree to which PCPs engage in this role for low-risk prostate cancer is unknown.

OBJECTIVE: Characterize PCP perceptions regarding their role in low-risk prostate cancer treatment decisionmaking.

DESIGN: Cross-sectional, national survey.

MAIN MEASURES: For men with low-risk prostate cancer, PCP reports of (1) confidence in treatment decisionmaking (high vs. low); (2) intended participation in key aspects of active surveillance treatment decision-making (more vs. less).

KEY RESULTS: A total of 347 from 741 eligible PCPs responded (adjusted response rate 56\%). Half of respondent PCPs (50.3\%) reported high confidence about engaging in low-risk prostate cancer treatment decision-making. The odds of PCPs reporting high confidence were greater among those in solo practice (vs working with $>1$ PCP) (OR 2.18; 95\% CI 1.14-4.17) and with higher volume of prostate cancer patients (> 15 vs. 6-10 in past year) (OR 2.16; 95\% CI 1.02-4.61). PCP report of their intended participation in key aspects of active surveillance treatment decision-making varied: discussing worry (62.4\%), reviewing benefits (48.5\%) and risks $(41.8 \%)$, and reviewing all treatment options (34.2\%). PCPs who reported high confidence had increased odds of more participation in all aspects of active surveillance decision-making: reviewing all treatment options (OR 3.11; 95\% CI 1.825.32), discussing worry (OR 2.12; 95\% CI 1.28-3.51), and reviewing benefits (OR 3.13; 95\% CI 1.89-5.16) and risks (OR 3.20; 95\% CI 1.91-5.36).

CONCLUSIONS: The majority of PCPs were confident about engaging with patients in low-risk prostate cancer treatment decision-making, though their intended participation varied widely across four key aspects of active surveillance care. With active surveillance being considered for other low-risk cancers (such as breast and thyroid), understanding factors influencing PCP involvement will be instrumental to supporting teambased cancer care.

Received January 24, 2020

Accepted October 14, 2020

Published online October 29, 2020
KEY WORDS: prostatic neoplasms; decision-making; primary care; watchful waiting.

J Gen Intern Med 36(2):447-54

DOI: $10.1007 / \mathrm{s} 11606-020-06318-8$

(C) Society of General Internal Medicine 2020

\section{INTRODUCTION}

The role of primary care providers (PCPs) in cancer care has expanded throughout the cancer continuum. Not only are there more patients who are diagnosed with and survivors of cancer, these patients also tend to be older and medically complex. This has led to PCPs managing more cancer care than just primary prevention. While PCP involvement during survivorship has flourished due to the increasing national emphasis on team-based cancer care delivery, recent research suggests that patients are involving their PCPs during the treatment stage. ${ }^{1-5}$ In particular, patients are discussing their initial cancer treatment options with their PCPs in addition to their cancer specialists (e.g., medical oncologist, radiation oncologist, or surgeon). Work by our team found that, in a population-based cohort of women with early-stage breast cancer, over a third of women and their PCPs reported participation by their PCP in their treatment decision-making.,

An area with great potential to leverage the role of PCPs is in the management of low-risk cancers. Due to the indolent nature of these cancers, treatment is evolving from surgery or radiation to active surveillance, which involves routine followup with clinical exams and lab tests and/or imaging. ${ }^{6-8}$ In fact, for patients with low-risk prostate cancer, multiple guidelines now recommend active surveillance as the primary management strategy. ${ }^{9,10}$ Because active surveillance involves management similar to any other chronic disease, it is well-aligned with PCP expertise. However, a key challenge in the implementation of active surveillance is uncertainty around which provider-urologist or $\mathrm{PCP}$ - should deliver the services required for this care. Prior research in cancer survivorship shows that, although PCPs desire involvement in their patient's ongoing care (such as monitoring for recurrence, providing psychosocial support, and symptom management), 
they often report lacking confidence and knowledge to do so. ${ }^{11-13}$ Confidence has been shown to be important for providers to engage effectively in disease management and care delivery. For example, clinicians reported the lack of knowledge and self-efficacy as barriers to adhering to clinical practice guidelines. ${ }^{14,15}$ Very little is known about how PCPs can help urologists deliver coordinated active surveillance care; thus, understanding PCP views - particularly their perceptions around their confidence and ability to engage in this care-is critical before expanding their roles into low-risk cancer management.

We therefore conducted a large national survey of PCPs to characterize their perspectives on their role in the management of low-risk prostate cancer. We explored PCP confidence to engage in treatment decision-making for men newly diagnosed with low-risk prostate cancer, and their intended participation when presented with a new patient on active surveillance. We then examined whether PCP confidence was associated with their participation in key aspects of treatment decision-making related to active surveillance.

\section{METHODS}

Using simple random sampling, we sent surveys to 1000 primary care physicians (internists, and family and general practitioners) from the American Medical Association Masterfile, a nationally representative database of physicians in the USA, between July and September 2018. Physicians were included if they responded on the survey to routinely providing care to men over 50 years. A $\$ 20$ unconditional incentive was provided with the first mailing. The mailing also included a link for physicians to complete the survey online. We used a modified Dillman method to maximize our response rate which involved follow-up to non-responders with a postcard reminder, phone call, and an additional mailing. ${ }^{16}$ The survey study at large aimed to examine PCP perspectives in the management of low-risk cancers; the data presented here represents a secondary data analysis.

\section{Measures}

Survey content was developed based on prior work, modifying measures used by our team on previous surveys, and pilot testing with urologists and PCPs. ${ }^{3,17,18}$

PCP Confidence in Treatment Decision-making. Respondents were asked to report on their overall confidence about engaging in treatment decision-making for men newly diagnosed with low-risk prostate cancer by responding to four statements: (1) "I have the knowledge to participate in treatment decision making," (2) "I am unsure what my role should be in treatment decision making," (3) "I have other priorities that limit my involvement in treatment decision making," and (4) "I feel confident in my ability to help with treatment decision making". Responses were on a 5-point Likert scale from 1 to 5 ("strongly disagree," "somewhat disagree," "neither agree nor disagree," "somewhat agree," "strongly agree"). Items "I am unsure what my role should be in treatment decision making" and "I have other priorities that limit my involvement in treatment decision making" were reverse recoded (e.g., "strongly disagree" was coded as 5 instead of 1 ). By doing so, higher scores on each statement corresponded to higher confidence. The four items were averaged to create a composite confidence scale, which was then validated using factor analysis. From this scale, we created a dichotomous variable, where physicians scoring over the median (3.5) were coded as having high confidence and those scoring 3.5 or less as having low confidence. We chose this categorization given our interest in PCPs' perception of having "high" confidence, a clinically meaningful concept for future interventions that could focus on improving PCP confidence. We thus used a conservative cut-off at the median which allowed us to capture PCPs who feel at least some level of confidence or more.

PCP Intended Participation in Treatment Decision-making. To assess how PCPs would intend to participate in treatment decision-making with a patient, they were presented with a clinical vignette of "Mr. Smith," a 68-year-old man with low-risk prostate cancer. The patient had discussed his treatment with his cancer specialists and was considering active surveillance as his management strategy. He was then seeing his PCP for his routine primary care follow-up visit. PCPs were asked to report what their level of participation would be regarding discussing four key aspects of treatment decision-making related to active surveillance. Respondents were asked how much time they would typically spend with Mr. Smith (1) reviewing all of his treatment options (surgery, radiation, active surveillance), (2) discussing any worries that he may have related to how to manage his cancer, (3) reviewing the risks of active surveillance such as progression of his cancer, and (4) reviewing the benefits of active surveillance such as avoiding side effects with surgery or radiation (e.g., urinary or bowel incontinence, erectile dysfunction). Responses were on a 5-point Likert scale from 1 to 5 ("not much," "little," "some," "much," or "a great deal") and dichotomized into less participation ("not much," "little," or "some" time spent) vs. more participation ("much" or "a great deal" time spent).

\section{Covariates}

PCP-reported characteristics collected via survey included gender, race, years since residency $(<5$ years ago, $5-$ 10 years ago, $\geq 10$ years ago), personal or family history of prostate cancer, volume of prostate cancer patients managed in the past year (low (0-5), medium (6-10), and high (>10)), and number of patients on active surveillance for their initial treatment in the past year (none (0), low (1-5), and high (> 5)). PCP-reported practice characteristics included academic affiliation, single vs. multi-specialty practice, and practice size (solo practitioner, $2-10,11-50,51$ or more). 


\section{Statistical Analysis}

Descriptive statistics were used to summarize physician and practice characteristics. We conducted a series of three analyses. First, distribution of PCP confidence to engage in low-risk prostate cancer treatment decision-making was generated. Using bivariate (chi-squared or $t$ tests as appropriate) followed by multivariable (logistic regression model) analyses, we examined associations between PCP confidence (high vs. low) and physician (gender, race, years since residency, personal or family history of prostate cancer, volume of prostate cancer patients, and number of patients on active surveillance) and practice (academic affiliation, single vs. multi-specialty practice, and practice size) characteristics. Second, we described PCP intended participation for the four key aspects of treatment decision-making related to active surveillance (reviewing all treatment options, discussing any worries that Mr. Smith may have, reviewing the risks of active surveillance, and reviewing the benefits of active surveillance). We then used bivariate and multivariable regression analyses to examine associations between PCP participation (less vs. more) for each aspect of active surveillance care and physician and practice characteristics (as detailed above). Third, we constructed four multivariable logistic regression models examining the association between PCP intended participation in treatment decision-making for each aspect of active surveillance care and PCP confidence to engage in low-risk prostate cancer treatment decision-making. Each model was adjusted for physician and practice characteristics (as detailed above). All analyses incorporated weights to reduce potential nonresponse bias. For non-respondents, we had data available on gender, age, specialty, and geographic region. Bivariate and multivariable comparisons of response rates indicated that lower response rates were significantly associated with northeast geographic region, and general practice and internal medicine specialties. Based on this, the propensity scores from a logistic model regressing survey response against specialty and geographic region were generated and the inverse of these scores were used as weights in our multivariable analyses.

We performed sensitivity analyses to ensure the robustness of our results. This included analyzing PCP confidence in treatment decision-making and PCP intended participation in treatment decision-making as continuous variables and using different cut points for the dichotomous variables. All analyses yielded largely similar results (data not shown).

Analyses were performed using SAS, version 9.4 (SAS Institute, Cary, NC). This study was deemed exempt by the University of Michigan Institutional Review Board.

\section{RESULTS}

In total, 347 of 741 eligible physicians responded to the survey (adjusted response rate 56\%) (Appendix Fig. 3). Physicians were excluded if they were not a PCP $(n=77)$, did not provide care to men $>50$ years $(n=66)$, for undeliverable mail $(n=$
83 ), or other reasons ( $n=11$; e.g., deceased). Table 1 shows physician and practice characteristics of respondents. PCPs were predominantly male (69.2\%), White (73.6\%), and graduated from residency 10 or more years ago $(93.4 \%)$. About a third of PCPs were in solo practices $(29.5 \%)$ and practices with academic affiliations (32.0\%). The majority of PCPs managed a low volume (0-5) of patients with prostate cancer in the last 12 months (44.1\%) and had low number (1-5) of patients on active surveillance (70.2\%).

\section{PCP Confidence in Treatment Decision-making}

Factor analysis indicated that the four items of the confidence scale represented a single factor, based on percentage of variance explained, and confirmed by a scree plot. The factor loadings were all sufficiently high with low variation (range 0.73-0.83), indicating that a score including all four items weighted equally is appropriate. Half of the PCPs (50.3\%)

Table 1 PCP-Reported Demographics and Practice Characteristics $(N=347)$

\begin{tabular}{|c|c|c|}
\hline & $N$ & $\%$ \\
\hline \multicolumn{3}{|l|}{ Gender } \\
\hline Male & 241 & 69.2 \\
\hline Female & 105 & 30.2 \\
\hline Unknown & & 0.6 \\
\hline \multicolumn{3}{|l|}{ Race } \\
\hline White & 256 & 73.6 \\
\hline Asian & 66 & 19.0 \\
\hline Black or African American & 11 & 3.2 \\
\hline American Indian or Alaskan Native & 4 & 1.2 \\
\hline Other & 11 & 3.2 \\
\hline \multicolumn{3}{|l|}{ Ethnicity } \\
\hline Not Hispanic or Latino & 313 & 89.9 \\
\hline Hispanic or Latino & 26 & 7.5 \\
\hline Unknown & 9 & 2.6 \\
\hline \multicolumn{3}{|l|}{ Specialty } \\
\hline Family Medicine & 146 & 42 \\
\hline General Practice & 109 & 31 \\
\hline Internal Medicine & 92 & 27 \\
\hline \multicolumn{3}{|l|}{ Graduation from residency } \\
\hline Less than 5 years ago & 9 & 2.8 \\
\hline At least 5 but less than 10 years ago & 12 & 3.8 \\
\hline 10 or more years ago & 297 & 93.4 \\
\hline \multicolumn{3}{|l|}{ Academic affiliation } \\
\hline No & 213 & 68.1 \\
\hline \multirow{2}{*}{\multicolumn{3}{|c|}{ Single vs. multi-specialty }} \\
\hline & & \\
\hline Single & 202 & 64.1 \\
\hline Multi & 113 & 35.9 \\
\hline \multicolumn{3}{|l|}{ Physicians at practice } \\
\hline 1 (solo practitioner) & 93 & 29.5 \\
\hline $2-10$ & 157 & 49.8 \\
\hline $11-50$ & 51 & 16.2 \\
\hline 51 or more & 14 & 4.4 \\
\hline \multicolumn{3}{|c|}{ Volume of men with prostate cancer managed in the last 12 months } \\
\hline Low $(0-5)$ & 152 & 44.1 \\
\hline Medium $(6-10)$ & 87 & 25.2 \\
\hline High (11+) & 106 & 30.7 \\
\hline \multicolumn{3}{|c|}{ Volume of men managed with AS in the last 12 months } \\
\hline None & 43 & 14.1 \\
\hline Low $(1-5)$ & 214 & 70.2 \\
\hline High $(6+)$ & 48 & 15.7 \\
\hline \multicolumn{3}{|l|}{ Personal history of prostate cancer } \\
\hline No & 298 & 96.1 \\
\hline Yes & 12 & 3.9 \\
\hline \multicolumn{3}{|l|}{ Family history of prostate cancer } \\
\hline No & 230 & 74.2 \\
\hline Yes & 80 & 25.8 \\
\hline
\end{tabular}


reported they had high confidence about engaging in treatment decision-making for men newly diagnosed with low-risk prostate cancer. Nearly two-thirds of PCPs somewhat or strongly agreed with statements indicating higher confidence: "I feel confident in my ability to help with treatment decision making" $(65 \%)$ and "I have the knowledge to participate in treatment decision making" (64\%). A third or less of PCPs somewhat or strongly agreed with statements indicating lower confidence: "I am unsure what my role should be in treatment decision making" (33\%) and "I have other priorities that limit my involvement in treatment decision making" (22\%) (Fig. 1) (distribution of responses in Appendix Table 3). Odds of reporting high confidence (vs. low) were greater for PCPs with a high volume of patients with prostate cancer (vs. medium volume in past 12 months) (odds ratio (OR) 2.16; 95\% confidence interval (CI) 1.02-4.61) and those in solo practice (vs. those who worked with more than 1 other PCP) (OR 2.18; 95\% CI 1.14-4.17).

\section{PCP Intended Participation in Treatment Decision-making}

There was variation in PCPs' intent to participate in different aspects of active surveillance treatment decision-making. Most frequently, PCPs reported more participation in discussing any worries Mr. Smith may have related to how to manage his cancer $(62.4 \%$; 95\% CI $57.2-67.6 \%)$. Just under half $(48.5 \%$; 95\% CI 43.2-53.9\%) reported more participation in reviewing the benefits of active surveillance and $41.8 \%$ (95\% CI 36.5-47.1\%) in reviewing the risks of active surveillance. Only $34.2 \%$ (95\% CI 29.1-39.3\%) reported more participation in reviewing all of Mr. Smith's treatment options (distribution of responses in Appendix Table 3). PCPs in solo practice reported more participation (vs. less) for reviewing all treatment options (OR 3.33; 95\% CI 1.81-6.14), for reviewing the risks (OR 2.77; 95\% CI 1.53-5.02), and for reviewing the benefits (OR 2.68; 95\% CI 1.49-4.84) of active surveillance (Table 2).

\section{Association Between PCP Intended Participation and PCP Confidence in Treatment Decision-making}

In adjusted models, PCPs who reported they had high confidence to engage in treatment decision-making for low-risk prostate cancer had increased odds of more participation in all four aspects of active surveillance treatment decision-making. This included increased odds of reviewing all treatment options (OR 3.11; 95\% CI 1.82-5.32), discussing any worries (OR 2.12; 95\% CI 1.28-3.51), reviewing the risks (OR 3.20; 95\% CI 1.91-5.36), and reviewing the benefits (OR 3.13; $95 \%$ CI 1.89-5.16) (Fig. 2).

\section{DISCUSSION}

Our study of a large, nationally representative sample of primary care physicians found that the majority of PCPs reported they had high confidence to engage in treatment decision-making for men newly diagnosed with low-risk prostate cancer. These PCPs were also more likely to report that they would participate more in several key aspects of treatment decision-making related to active surveillance including reviewing all treatment options, discussing patient worry, and reviewing the risks and benefits of active surveillance.

To our knowledge, our results are the first to highlight the potential expanding role for PCPs in the decision-making

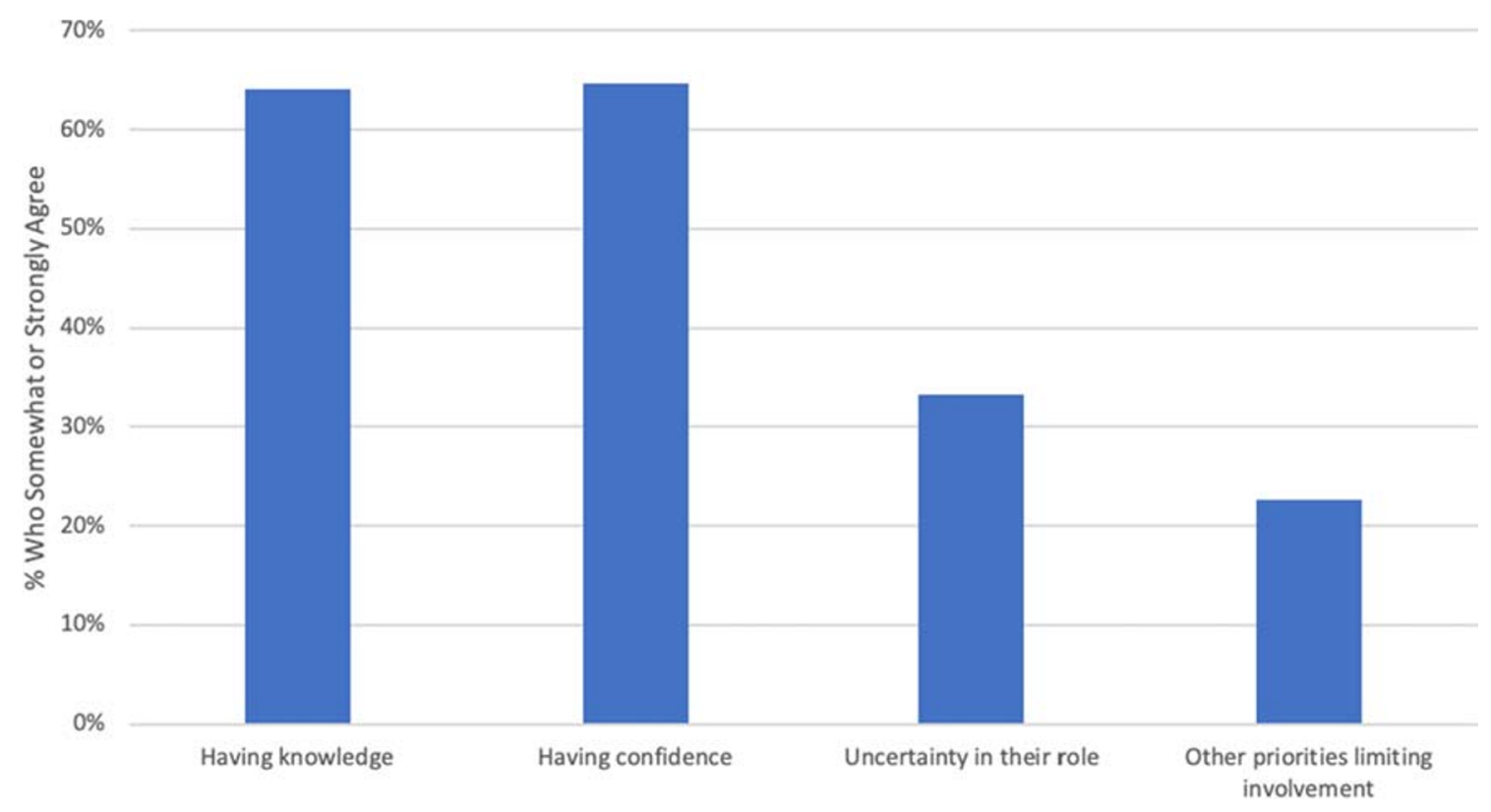

Fig. 1 Percent of PCPs who somewhat or strongly agree with four statements comprising PCP confidence to engage in treatment decisionmaking for men newly diagnosed with low-risk prostate cancer. 
Table 2 Multivariable Odds Ratios and 95\% Confidence Intervals of Physician and Practice Characteristics Associated with PCP Intended Participation in Four Key Aspects of Treatment Decision-making Related to Active Surveillance Care

\begin{tabular}{|c|c|c|c|c|}
\hline & $\begin{array}{l}\text { Reviewing all treatment } \\
\text { options, OR (95\% CI) }\end{array}$ & $\begin{array}{l}\text { Discussing worry, } \\
\text { OR (95\% CI) }\end{array}$ & $\begin{array}{l}\text { Reviewing active surveillance } \\
\text { risks, OR (95\% CI) }\end{array}$ & $\begin{array}{l}\text { Reviewing active surveillance } \\
\text { benefits, OR (95\% CI) }\end{array}$ \\
\hline \multicolumn{5}{|c|}{ Volume of men with prostate cancer managed in the last 12 months } \\
\hline $\begin{array}{l}\text { Low }(0-5) \text { vs. } \\
\text { medium }(6-10)\end{array}$ & $1.02(0.54,1.94)$ & $1.22(0.65,2.27)$ & $1.30(0.70,2.40)$ & $0.90(0.49,1.65)$ \\
\hline $\begin{array}{l}\text { High }(11+) \text { vs. } \\
\text { medium }(6-10)\end{array}$ & $1.11(0.57,2.17)$ & $1.03(0.53,1.97)$ & $0.90(0.47,1.73)$ & $0.79(0.42,1.49)$ \\
\hline \multicolumn{5}{|c|}{ Volume of men managed with $\mathrm{AS}$ in the last 12 months } \\
\hline Low $(1-5)$ vs. none & $1.03(0.48,2.22)$ & $1.22(0.59,2.54)$ & $1.01(0.48,2.11)$ & $1.29(0.62,2.67)$ \\
\hline High (6+) vs. none & $1.25(0.45,3.46)$ & $1.13(0.42,3.05)$ & $1.80(0.67,4.84)$ & $1.38(0.52,3.67)$ \\
\hline \multicolumn{5}{|l|}{ Academic affiliation } \\
\hline Yes vs. no & $1.03(0.59,1.78)$ & $0.73(0.43,1.25)$ & $1.07(0.63,1.81)$ & $1.26(0.75,2.11)$ \\
\hline $\begin{array}{l}\text { Solo practitioner } \\
\text { Yes vs. no }\end{array}$ & $3.33(1.81,6.14)$ & $1.43(0.77,2.65)$ & $2.77(1.53,5.02)$ & $2.68(1.49,4.84)$ \\
\hline \multicolumn{5}{|l|}{ Specialty } \\
\hline Multi vs. single & $0.99(0.56,1.76)$ & $0.63(0.36,1.08)$ & $0.96(0.56,1.65)$ & $0.98(0.58,1.66)$ \\
\hline \multicolumn{5}{|c|}{ Graduation from residency } \\
\hline $\begin{array}{l}10+\text { years vs. }< \\
5 \text { years }\end{array}$ & $1.47(0.29,7.43)$ & $1.00(0.27,3.75)$ & $0.50(0.14,1.83)$ & $0.85(0.24,3.04)$ \\
\hline $\begin{array}{l}5-9 \text { years vs. }< \\
5 \text { years }\end{array}$ & $3.56(0.54,23.44)$ & $0.60(0.12,3.16)$ & $0.34(0.06,1.84)$ & $0.51(0.10,2.69)$ \\
\hline \multicolumn{5}{|l|}{ Gender } \\
\hline $\begin{array}{l}\text { Female vs. male } \\
\text { Race }\end{array}$ & $0.84(0.48,1.47)$ & $0.89(0.51,1.54)$ & $0.57(0.33,0.99)$ & $0.59(0.35,0.10)$ \\
\hline $\begin{array}{l}\text { American Indian vs. } \\
\text { White }\end{array}$ & $0.43(0.04,4.62)$ & $0.11(0.01,1.20)$ & $0.79(0.10,6.16)$ & $0.75(0.10,5.71)$ \\
\hline Asian vs. White & $1.66(0.87,3.14)$ & $0.79(0.43,1.47)$ & $1.20(0.64,2.23)$ & $1.82(0.98,3.39)$ \\
\hline Black vs. White & $1.14(0.31,4.26)$ & $5.91(0.690,50.73)$ & $1.74(0.48,6.32)$ & $0.97(0.27,3.49)$ \\
\hline Latino vs. White & $1.61(0.59,4.38)$ & $1.57(0.55,4.49)$ & $0.71(0.26,1.91)$ & $2.50(0.91,6.88)$ \\
\hline Other vs. White & $2.74(0.73,10.37)$ & $9.48(1.11,80.92)$ & $2.15(0.57,8.21)$ & $3.05(0.79,11.83)$ \\
\hline
\end{tabular}

Italicized values signify statistically significant ORs at $p<0.05$

about low-risk cancer treatment, specifically those where active surveillance is an option. Among PCPs who treat women with early-stage breast cancer, we previously found that a third reported involvement in their patient's breast cancer treatment decisions, including surgery, radiation, and chemotherapy decisions. ${ }^{3}$ In the cancer survivorship literature, it has often been reported that PCPs lack confidence or the knowledge and skills to be able to participate in cancer care. ${ }^{19,20}$ In a survey of PCPs regarding colon and breast cancer survivor care, less than half of the PCPs reported feeling very confident in their knowledge about testing for recurrence, caring for late physical effects and psychosocial effects. ${ }^{21}$ However, nearly twothirds of respondents from our survey perceived having the knowledge and the ability to participate in treatment decisionmaking for low-risk prostate cancer. This may reflect the increasing population of men with low-risk prostate cancer that are invariably also seen and managed in primary care; supporting this, physicians in our cohort who had larger volumes of prostate cancer patients were more likely to report they had high confidence to engage in treatment decision-

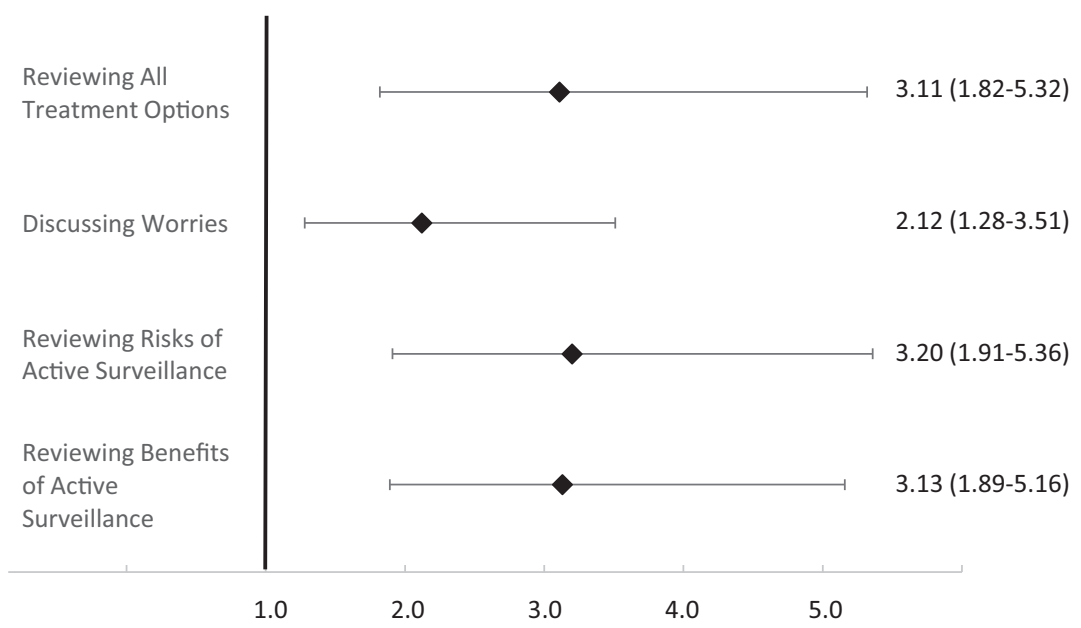

Fig. 2 Adjusted odds ratio* and 95\% confidence intervals of PCP intended participation in treatment decision-making for active surveillance care associated with PCP confidence in treatment decision-making for men with low-risk prostate cancer. *Adjusted for physician (gender, race, years since residency, personal or family history of prostate cancer, volume of prostate cancer patients, and number of patients on active surveillance) and practice (academic affiliation, single vs. multi-specialty practice, and practice size) characteristics. 
making. This generally high confidence held by PCPs in lowrisk prostate cancer management is promising for the eventual success of team-based care models incorporating PCPs.

In our sample, compared to PCPs in multi-physician practices, PCPs in solo practice reported they had high confidence to engage in low-risk prostate cancer treatment decision-making and were more likely to participate in the key aspects of active surveillance treatment decision-making. PCPs in solo practices may have longer relationships with their patients and feel more comfortable engaging in these aspects of their care. This may also reflect the organizational set-up of practices; compared to solo practitioners, those in multi-physician practices may be more likely to have multidisciplinary care, enabling their patients to connect more easily with specialists. PCPs may not then engage in cancer treatment decision-making, and rather defer to the cancer specialist to handle this care. However, in light of increasing number of low-risk cancer patients with more considering active surveillance and cancer specialist workforce shortages, PCP involvement may be necessary if not inevitable. While the influence of organizational factors in the diffusion of an innovation has been well characterized, further research is needed to understand how a health care system can make changes to encourage their PCPs to engage in cancer care (including treatment). ${ }^{22}$

It is not surprising that PCPs who reported having high confidence to engage in treatment decision-making for lowrisk prostate cancer often reported participating more in key aspects of treatment decision-making for active surveillance. Yet, more reassuring is finding that PCPs were equally more likely to review the benefits of active surveillance as they were the risks. Giving equal weight to both sides of a treatment choice is a key component of informed decision-making, yet one that is not always present. For instance, prior literature has shown that physicians order tests or medications because they believe that is what the patient wants, even if it is not clinically appropriate (e.g., prescribing antibiotics for viral infections, continuing cancer screening beyond screening age recommendations) ${ }^{23-25}$ Because some men with low-risk prostate cancer believe active surveillance is "doing nothing," and report anxiety with making this choice, it is plausible that PCPs may be hesitant to discuss the benefits of active surveillance, perceiving it as "going against" what a patient may want. ${ }^{26,27}$ Our results however suggest that PCPs are not limiting their treatment discussions which is critical for the continued success of active surveillance for low-risk prostate cancer management.

Strengths of this study include a nationally representative population of PCPs with a robust response rate. However, there are potential limitations to acknowledge. First, we probed PCPs' beliefs about actual participation using a clinical vignette; this may be subject to social desirability bias with PCPs reporting on how they should versus how they actually practice. Second, PCPs reported their participation in the patient's initial stages of treatment decision-making. Cancer treatment decision-making is often a dynamic process and, in the case of low-risk prostate cancer, may evolve over time with men choosing to receive definitive treatment after initially starting active surveillance. Therefore, PCP participation may change over time. Third, though we achieved a $56 \%$ response rate for a physician survey, it is possible that non-response bias may have affected our findings. We accounted for this by incorporating weights in our analyses to reduce potential non-response bias. Fourth, our study is cross-sectional and therefore inferences regarding causality are limited. Additional studies that help to delineate whether perceived confidence influences PCP's participation in treatment discussion or rather participation in treatment discussion influences confidence are needed.

\section{CONCLUSION}

Our findings augment existing literature about the role of primary care providers in cancer care. These results suggest a role for PCPs much earlier in the cancer treatment continuum, specifically in supporting treatment decision-making for their patients with low-risk prostate cancer. While active surveillance is the guideline recommended strategy for low-risk prostate cancer, it is increasingly being considered for the management of other low-risk cancers, such as breast and thyroid. ${ }^{28-30}$ Future studies that provide granularity in factors influencing PCP involvement and their ability to provide coordinated care will be critical towards developing interventions to improve team-based care for low-risk cancer management.

Corresponding Author: Archana Radhakrishnan, MD, MHS; Department of Internal Medicine, University of Michigan, Ann Arbor, MI, USA (e-mail: arra@med.umich.edu).

Funding Funding for this research was received from an institutional research grant from the University of Michigan Rogel Cancer Center.

\section{Compliance with Ethical Standards:}

Conflict of Interest: The authors declare that they do not have a conflict of interest. 


\section{APPENDIX}

\section{PCPs identified through AMA}

for mailing; $\mathrm{N}=1000$

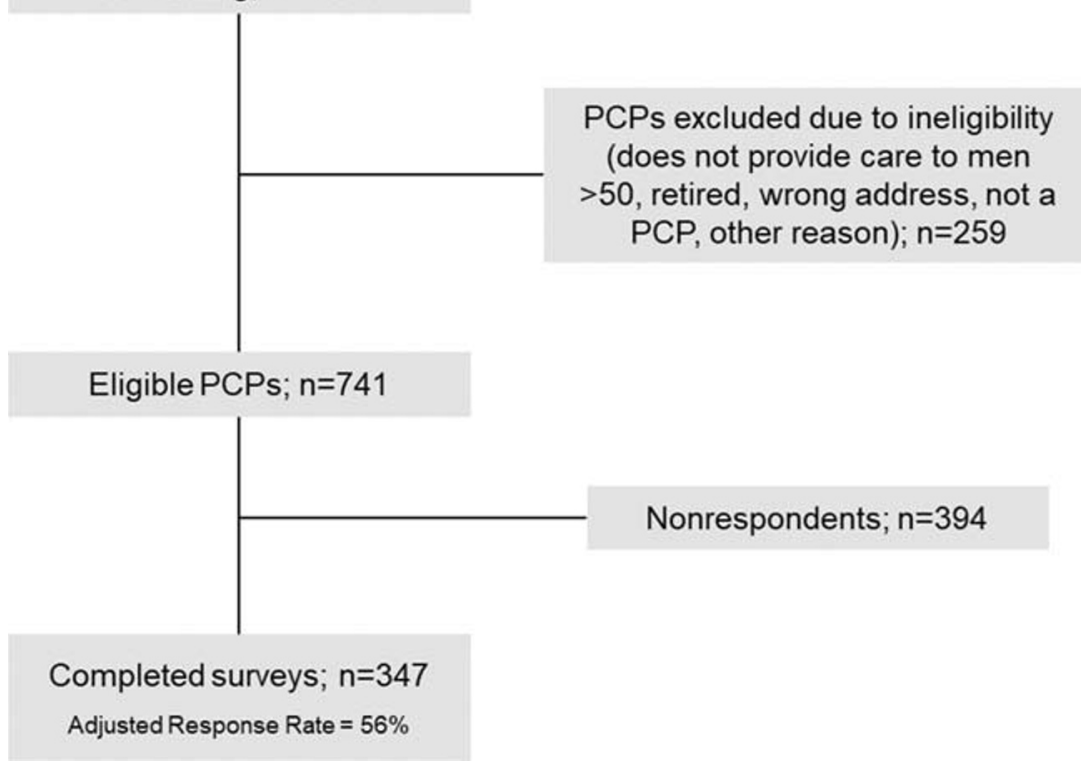

Fig. 3 Flow diagram demonstrating study cohort

Table 3 Distribution of Responses for PCP Confidence in Treatment Decision-making and PCP Intended Participation in Treatment Decisionmaking

\begin{tabular}{|c|c|c|c|c|c|c|c|c|}
\hline \multicolumn{9}{|c|}{ For men newly diagnosed with low-risk prostate cancer making a treatment decision... } \\
\hline & \multicolumn{2}{|c|}{$\begin{array}{l}\text { I have the } \\
\text { knowledge to } \\
\text { participate in } \\
\text { treatment } \\
\text { decision-making } \\
(N, \%)\end{array}$} & \multicolumn{2}{|c|}{$\begin{array}{l}\text { I am unsure what } \\
\text { my role should be } \\
\text { in treatment } \\
\text { decision-making } \\
(N, \%)\end{array}$} & \multicolumn{2}{|c|}{$\begin{array}{l}\text { I have other } \\
\text { priorities that } \\
\text { limit my } \\
\text { involvement in } \\
\text { treatment } \\
\text { decision-making } \\
(N \%)\end{array}$} & \multicolumn{2}{|c|}{$\begin{array}{l}\text { I feel confident in } \\
\text { my ability to help } \\
\text { with treatment } \\
\text { decision-making } \\
(N, \%)\end{array}$} \\
\hline Strongly disagree & 12 & $4 \%$ & 85 & $25 \%$ & 113 & $34 \%$ & 17 & $5 \%$ \\
\hline Somewhat disagree & 54 & $16 \%$ & 94 & $28 \%$ & 77 & $23 \%$ & 53 & $16 \%$ \\
\hline Neither agree nor disagree & 55 & $16 \%$ & 46 & $14 \%$ & 69 & $21 \%$ & 50 & $15 \%$ \\
\hline Somewhat agree & 142 & $42 \%$ & 88 & $26 \%$ & 59 & $18 \%$ & 140 & $41 \%$ \\
\hline Strongly agree & 74 & $22 \%$ & 24 & $7 \%$ & 17 & $5 \%$ & 80 & $24 \%$ \\
\hline \multicolumn{9}{|c|}{$\begin{array}{l}\text { How much time would you typically spend with Mr. Smith... } \\
\qquad \begin{array}{l}\text { Reviewing all of } \\
\text { his treatment } \\
\text { options (surgery, } \\
\text { radiation, active } \\
\text { surveillance) }\end{array}\end{array}$} \\
\hline Not much & 29 & $9 \%$ & 6 & $2 \%$ & 25 & $7 \%$ & 17 & $5 \%$ \\
\hline Little & 38 & $11 \%$ & 13 & $4 \%$ & 35 & $10 \%$ & 26 & $8 \%$ \\
\hline Some & 156 & $46 \%$ & 108 & $32 \%$ & 136 & $40 \%$ & 132 & $39 \%$ \\
\hline Much & 88 & $26 \%$ & 152 & $45 \%$ & 111 & $33 \%$ & 124 & $36 \%$ \\
\hline A great deal & 28 & $8 \%$ & 59 & $17 \%$ & 30 & $9 \%$ & 41 & $12 \%$ \\
\hline
\end{tabular}




\section{REFERENCES}

1. McCabe MS, Bhatia S, Oeffinger KC, et al. American Society of Clinical Oncology statement: achieving high-quality cancer survivorship care. J Clin Oncol 2013;31(5):631-640.

2. Radhakrishnan A, Grande D, Ross M, et al. When primary care providers (PCPs) help patients choose prostate cancer treatment. J Am Board Fam Med 2017;30(3):298-307.

3. Wallner LP, Li Y, McLeod MC, et al. Primary care provider-reported involvement in breast cancer treatment decisions. Cancer. 2019;125(11): 1815-1822.

4. Wallner LP, Abrahamse P, Uppal JK, et al. Involvement of primary care physicians in the decision making and care of patients with breast cancer. J Clin Oncol 2016;34(33):3969-3975.

5. National Research Council, Institute of Medicine. From Cancer Patient to Cancer Survivor: Lost in Transition. 2005.

6. Cooperberg MR, Carroll PR. Trends in management for patients with localized prostate cancer, 1990-2013. JAMA. 2015;314(1):80-82.

7. Loeb S, Byrne N, Makarov DV, Lepor $\mathbf{H}$, Walter D. Use of conservative management for low-risk prostate cancer in the Veterans affairs integrated health care system from 2005-2015. JAMA. 2018;319(21):2231-2233.

8. Mahal BA, Butler S, Franco I, et al. Use of active surveillance or watchful waiting for low-risk prostate cancer and management trends across risk groups in the United States, 2010-2015. JAMA. 2019;321(7):704-706.

9. Chen RC, Rumble RB, Loblaw DA, et al. Active surveillance for the management of localized prostate cancer (Cancer Care Ontario Guideline): American Society of Clinical Oncology clinical practice guideline endorsement. J Clin Oncol 2016;34(18):2182-2190.

10. Sanda MG, Chen RC, Crispino T, et al. Clinically localized prostate cancer: AUA/ASTRO/SUO guideline. American Urological Association. 2017; 2017.

11. Lawrence RA, McLoone JK, Wakefield CE, Cohn RJ. Primary care physicians' perspectives of their role in cancer care: a systematic review. J Gen Intern Med 2016;31(10):1222-1236.

12. Dossett LA, Hudson JN, Morris AM, et al. The primary care provider (PCP)-cancer specialist relationship: a systematic review and mixedmethods meta-synthesis. CA Cancer J Clin 2017;67(2):156-169.

13. Rubinstein EB, Miller WL, Hudson SV, et al. Cancer survivorship care in advanced primary care practices: a qualitative study of challenges and opportunities. JAMA Intern Med 2017;177(12):1726-1732.

14. Cabana MD, Rand CS, Powe NR, et al. Why don't physicians follow clinical practice guidelines?A framework for improvement. JAMA. 1999;282(15): 1458-1465.

15. Hall AM, Scurrey SR, Pike AE, et al. Physician-reported barriers to using evidence-based recommendations for low back pain in clinical practice: a systematic review and synthesis of qualitative studies using the Theoretical Domains Framework. Implement Sci 2019;14(1):49.

16. Dillman DA, Smyth JD, Christian LM. Internet, Phone, Mail, and Mixed-Mode Surveys: The Tailored Design Method. Hoboken: Wiley Publishing; 2014.
17. Jagsi R, Huang G, Griffith $\mathbf{K}$, et al. Attitudes toward and use of cancer management guidelines in a national sample of medical oncologists and surgeons. J Natl Compr Cancer Netw 2014;12(2):204-212.

18. Survey of Physician Attitudes Regarding the Care of Cancer Survivors (SPARCCS). National Institutes of Health/American Cancer Society. Published 2009. Accessed 2018.

19. Virgo KS, Lerro CC, Klabunde CN, Earle C, Ganz PA. Barriers to breast and colorectal cancer survivorship care: perceptions of primary care physicians and medical oncologists in the United States. J Clin Oncol 2013;31(18):2322-2336.

20. Cheung WY, Aziz N, Noone AM, et al. Physician preferences and attitudes regarding different models of cancer survivorship care: a comparison of primary care providers and oncologists. J Cancer Surviv 2013;7(3):343-354.

21. Potosky AL, Han PK, Rowland J, et al. Differences between primary care physicians' and oncologists' knowledge, attitudes and practices regarding the care of cancer survivors. J Gen Intern Med 2011;26(12):1403-1410.

22. Berwick DM. Disseminating innovations in health care. JAMA. 2003;289(15):1969-1975.

23. Schoenborn NL, Boyd CM, Lee SJ, Cayea D, Pollack CE. Communicating about stopping cancer screening: comparing clinicians' and older adults' perspectives. Gerontologist. 2019;59(Suppl 1):S67-s76.

24. Linder JA, Singer DE. Desire for antibiotics and antibiotic prescribing for adults with upper respiratory tract infections. J Gen Intern Med 2003; 18(10):795-801.

25. Schoenborn NL, Bowman TL 2nd, Cayea D, Pollack CE, Feeser S, Boyd C. Primary care practitioners' views on incorporating long-term prognosis in the care of older adults. JAMA Intern Med 2016;176(5):671678.

26. Xu J, Neale AV, Dailey RK, Eggly S, Schwartz KL. Patient perspective on watchful waiting/active surveillance for localized prostate cancer. J Am Board Fam Med 2012;25(6):763-770.

27. Mader EM, Li HH, Lyons KD, et al. Qualitative insights into how men with low-risk prostate cancer choosing active surveillance negotiate stress and uncertainty. BMC Urol 2017;17(1):35.

28. Haymart MR, Miller DC, Hawley ST. Active surveillance for low-risk cancers - a viable solution to overtreatment? N Engl J Med 2017;377(3):203-206.

29. Brito JP, Hay ID, Morris JC. Low risk papillary thyroid cancer. Br Med J 2014;348:g3045.

30. Hwang ES, Hyslop T, Lynch T, et al. The COMET (Comparison of Operative versus Monitoring and Endocrine Therapy) trial: a phase III randomised controlled clinical trial for low-risk ductal carcinoma in situ (DCIS). BMJ Open 2019;9(3):e026797.

Publisher's Note: Springer Nature remains neutral with regard to jurisdictional claims in published maps and institutional affiliations. 